\title{
Influence of Roadway Cross-Section Shape on Gas Explosion Shock Wave Law in U-Type Ventilation Working Faces
}

\author{
Jiajia Liu $(\mathbb{D}),{ }^{1,2,3}$ Mengqi Shen $\left(\mathbb{D},{ }^{1}\right.$ Shouqi Chen $\mathbb{D D}^{1}$ and Ming Yang $\mathbb{D}^{1,2,3}$ \\ ${ }^{1}$ School of Safety Science and Engineering, Henan Polytechnic University, Jiaozuo 454000, China \\ ${ }^{2}$ Collaborative Innovation Center of Coal Work Safety and Clean-Efficiency Utilization, Jiaozuo 454000, China \\ ${ }^{3}$ State Key Laboratory Cultivation Base for Gas Geology and Gas Control, Henan Polytechnic University, Jiaozuo 454000, China
}

Correspondence should be addressed to Jiajia Liu; liujiajia@hpu.edu.cn

Received 20 May 2021; Revised 10 November 2021; Accepted 29 November 2021; Published 18 December 2021

Academic Editor: Jianwei Cheng

Copyright (c) 2021 Jiajia Liu et al. This is an open access article distributed under the Creative Commons Attribution License, which permits unrestricted use, distribution, and reproduction in any medium, provided the original work is properly cited.

\begin{abstract}
In U-shaped ventilation working face, different tunnel section shapes are one of the important factors affecting the propagation of gas explosion shock wave. In order to study the propagation law of gas explosion shock wave in working face, the numerical simulation study was carried out by using Fluent simulation software combined with the actual situation of gas explosion in \#415 working face of Chenjiashan Coal Mine in Shaanxi Province. By constructing a three-dimensional mathematical and physical model, a simulation study of the upper-corner gas explosion was carried out. The results are described as follows. (1) After the gas explosion shock wave propagates $40 \mathrm{~m}$, the overpressure peak equidistant difference tends to be stable and attenuates and propagates in the form of a single shock wave. The study determines that the effective length of the U-shaped ventilation inlet/ return tunnel is $40 \mathrm{~m}$. (2) When the tunnel section is trapezoidal, the initial overpressure of the gas explosion shock wave propagating to the inlet/return airway is the highest, followed by rectangular and semicircular arches, but the internal overpressure attenuation trend of different cross-sectional shapes is the same. (3) The gas explosion shock wave propagates radially along the working face section during the working face propagation. The farther away the location is from the upper corner of the tunnel during a gas explosion with different cross-sectional shapes, the closer the cutoff overpressure peak is. The attenuation trend of overpressure with the propagation distance conforms to the power function law. The research results provide an important theoretical direction for the numerical simulation of gas explosions in coal mining faces.
\end{abstract}

\section{Introduction}

With the increase in coal mining depth, underground gas emission and gas accumulation are becoming more and more serious. The upper corner of a coal mining face is the hazardous area of gas accumulation in coal mine and is the hot spot for gas explosion accidents in coal mines. After the gas explosion accident in upper corner, the huge gas explosion shock wave will spread along the inlet and outlet tunnel, causing serious damage to underground facilities and miners.

The propagation process of explosion shock wave is a process of constant conversion between energy and the external environment. In this process, as the energy of explosion shock wave is gradually converted into gas energy, the propagation length of explosion shock wave is limited. Therefore, studying the effective length can determine the energy dissipation capacity of explosion shock wave propagation. In addition, the cross-section shape is also one of the factors affecting the propagation of the blast wave. Different cross-section shapes have an impact on the propagation speed of the blast wave and the external energy conversion capacity.

Scholars have conducted a significant amount of research on the propagation law of gas explosion shock waves. Both et al. adjusted and optimized the grid model parameters of turbulence and combustion by simulating gas explosions and significantly improved the prediction of the development model [1]. Jing et al. deduced the relationship between the peak value of overpressure and the gas velocity 
and propagation distance in the process of a gas explosion [2]. Some scholars studied the influence of various conditions (initial temperature, ignition conditions, gas concentration, and barrier type) on gas explosion parameters [3-12]. Some scholars obtained the mechanism of suppressing a gas explosion by ultrasonic water mist and a vacuum chamber through experiments and numerical simulations $[13,14]$.

Some scholars studied the distribution and propagation law of gas explosion shock waves in different media of pipelines [15-18]. Some scholars studied the influences of pipe length, pipe diameter, and space distance on gas explosion overpressure [19-21]. Li and Hao simulated the ignition and propagation characteristics of gas explosions by introducing basic chemical reactions [22]. Some scholars studied the propagation law of gas explosion shock waves in roadways with different turning angles and on bifurcated roadways [23-27]. Some scholars simulated and studied the propagation law of gas explosion shock waves in the underground parallel roadway network $[28,29]$. Some scholars simulated and analyzed the propagation law of gas explosion shock waves in coal mining face roadways $[30,31]$.

Many scholars have focused on the pipeline shock wave propagation law and its influencing factors in gas explosion studies, but the actual tunnel effective length and cross-section shape impacts of the gas explosion shock wave law have not been reported. The explosion energy generated by gas accumulation under different geological conditions and different gas pressures is different, so the effective length of blast wave propagation is also different. Studying the effective length of blast wave propagation is beneficial to determine the energy exchange ability of the energy generated by explosion with the outside world. Therefore, the author uses Fluent simulation software, combined with the actual situation of a gas explosion in the upper corner of the \#415 working face in Chenjiashan coal mine, Shaanxi Province. To determine the effective length of the air inlet/return roadway, a numerical simulation of an upper-corner gas explosion of different cross-sectional shapes of a U-type ventilation roadway is carried out. The research results provide theoretical guidance for the prevention and control of gas explosion disasters in coal mining faces.

\section{Construction and Verification of Mathematical Model}

2.1. Basic Assumptions and Boundary Conditions of Numerical Simulation. Gas explosion is a very complex fluid elasticplastic process, accompanied by chemical reaction, turbulence changes, and other phenomena, so it is difficult to simulate the law of mine gas explosion after considering all conditions. In order to simulate the law of mine gas explosion as much as possible and ensure the reliability of numerical simulation of gas explosion, some basic assumptions must be made:

(1) Both premixed gas and combustion products satisfy the ideal gas state equation

(2) The specific heat capacity of the mixed gas follows the mixing rule, and the specific heat capacity of each component is a function of temperature

(3) The wall of the physical model is a rigid adiabatic wall, and there is no relative displacement

(4) The gas explosion reaction is one-way and irreversible

(5) In the physical model, the gas filling area is a normal uniform mixture of gas and air, and it is an ideal state

The model parameters and boundary conditions of gas explosion numerical simulation are as follows. The boundary conditions: the pipe boundary is set as the adiabatic wall with the temperature of $300 \mathrm{~K}$, and the outlet above the vertical pipe is set as the pressure outlet.

Basic model parameter: the initial conditions of the burned zone are $T=1600 \mathrm{~K}, \mathrm{H}_{2} \mathrm{O}$ volume fraction is 0.118 , $\mathrm{CO}_{2}$ volume fraction is 0.145 , and initial pressure is $101325 \mathrm{~Pa}$; the initial conditions of unburned zone are as follows: $T=300 \mathrm{~K}, \mathrm{CH}_{4}$ volume fraction is $0.053, \mathrm{O}_{2}$ volume fraction is 0.21 , and $\mathrm{H}_{2} \mathrm{O}$ and $\mathrm{CO}_{2}$ volume fraction is 0 ; the initial conditions of the air zone are as follows: the volume fraction of $\mathrm{CH}_{4}, \mathrm{H}_{2} \mathrm{O}$, and $\mathrm{CO}_{2}$ is 0 and the volume fraction of $\mathrm{O}_{2}$ is 0.233 .

\subsection{Construction of Gas Explosion Mathematical Model.} A gas explosion is a combustion reaction process, and the reaction is rapid. Assuming that the mixture of methane and air is an ideal gas, the hydrodynamic mathematical model of methane gas is established without considering the unstable factors such as viscosity, heat conduction, diffusion, and detonation; the conservation equation of the process is described as follows.

Equation of state is as follows:

$$
\begin{aligned}
p & =p(\rho, T) \\
& =\rho R T .
\end{aligned}
$$

Continuity equation is as follows:

$$
\frac{\partial \rho}{\partial t}+\frac{\partial(\rho u)}{\partial x}+\frac{\partial(\rho v)}{\partial y}+\frac{\partial(\rho w)}{\partial z}=0
$$

Energy equation is as follows:

$$
\frac{\partial}{\partial t}(\rho e)+u \frac{\partial(\rho e+p)}{\partial x}+v \frac{\partial(\rho e+p)}{\partial y}+w \frac{\partial(\rho e+p)}{\partial z}=0
$$

Momentum equation is as follows: 


$$
\left\{\begin{array}{l}
\frac{\partial u}{\partial t}+u \frac{\partial u}{\partial x}+v \frac{\partial u}{\partial y}+w \frac{\partial u}{\partial z}=-\frac{1}{p} \frac{\partial p}{\partial x} \\
\frac{\partial v}{\partial t}+u \frac{\partial v}{\partial x}+v \frac{\partial v}{\partial y}+w \frac{\partial v}{\partial z}=-\frac{1}{p} \frac{\partial p}{\partial y} \\
\frac{\partial w}{\partial t}+u \frac{\partial w}{\partial x}+v \frac{\partial w}{\partial y}+w \frac{\partial w}{\partial z}=-\frac{1}{p} \frac{\partial p}{\partial z}
\end{array}\right.
$$

where $P$ is the pressure; $t$ is the time; $x, y$, and $z$ are rectangular coordinate system parameters; $u, V$, and $W$ are the velocities in three coordinate directions; $\rho$ is the fluid density; $T$ is the temperature; $R$ is the gas constant; $e$ is the specific energy; and $e=p /(\gamma-1)+\rho\left(u^{2}+v^{2}+w^{2}\right) / 2(\gamma$ is the gas index).

\subsection{Turbulence Model and Combustion Model. Gas explo-} sion will lead to turbulent combustion, so the turbulence model should be selected in numerical simulation, and the correlation terms and turbulence viscosity coefficients can be calculated by constructing differential equations. According to the number of differential equations required for solution, the turbulence model can be divided into zero equation model, single equation model, and two-side equation model [32]. The large eddy numerical model can better simulate the required results by referring to the literature and several times of practical calculation and debugging [33-35]. Therefore, the LES turbulence model is used in this paper to describe the characteristics of turbulent flow field in combustion process.

Chemical reaction rates can be calculated using four models: laminar finite rate model, laminar finite rate/vortex dissipation model, vortex diffusion model, and vortex diffusion conceptual model.

The laminar flow finite velocity model ignores the influence of turbulent pulsation and uses Arrhenius to calculate the combustion rate. The condition is laminar flame, which is not suitable for turbulent combustion. The laminar finite velocity/eddy current dissipation model is characterized by the fact that the reaction rate is smaller than Arrhenius and eddy current dissipation equation, which is suitable for single-stage and two-stage reaction mechanism but not for multistage reaction mechanism. The reaction rate of the eddy current dissipation model is controlled by turbulent mixing for non-premixed flames. Combining the advantages and disadvantages of the four models, the vortex dissipation vortex diffusion model suitable for the LES turbulence model is adopted in this paper.

2.4. Verification of Calculation Model. According to previous gas explosion experiments in pipelines [36], a full-scale simulation comparison of $1: 1$ was carried out using Fluent simulation software to verify the reliability of the large eddy simulation turbulence equation and eddy-dissipation vortex diffusion model. In the test, the width and height of the straight pipe are both $80 \mathrm{~mm}$, the length is $19.2 \mathrm{~m}$, and the length of the vertical pipe is $5 \mathrm{~m}$. According to the setting of the experimental monitoring points, measuring point 1 is $19 \mathrm{~m}$ away from the leftmost end and is located on the central line of the horizontal pipeline, and measuring point 2 is located $0.5 \mathrm{~m}$ away from the central line of the horizontal pipeline. A section diagram of the physical model and the locations of the measuring points are shown in Figure 1. The left end of the pipeline is closed and filled with gas, and the pressure outlet is above the vertical pipeline.

The initial conditions and boundary conditions are consistent with those in the gas explosion experiment [37]; specifically, the gas concentration in the gas filling area is $0.053 ; \mathrm{O}_{2}$ concentration is $0.212 ; \mathrm{CO}_{2}$ concentration in the air area is 0.1456; and $\mathrm{H}_{2} \mathrm{O}$ concentration is 0.11925 . The pipeline boundary is set as an adiabatic wall, the temperature is $300 \mathrm{~K}$, and the outlet above the vertical pipe is set as the pressure outlet.

The selected calculation model is as follows: the fluid flow is unsteady turbulent flow, the turbulence model adopts the large eddy simulation turbulence equation, the wall adopts a standard wall function, the chemical reaction of methane combustion adopts an eddy-dissipation vortex diffusion model that is suitable for turbulent combustion, and a simple algorithm is used for an iterative solution. The simple algorithm is a widely used numerical method to solve flow field in computational fluid mechanics. It can not only solve nonlinear problems but also complete the coupling of velocity and pressure. Therefore, the simple algorithm is used for iterative solution.

The experimental and simulation results are shown in Figure 2.

As can be seen from Figure 2, in measuring point 1, when the inflatable length is, respectively, $4 \mathrm{~m}, 5.5 \mathrm{~m}$, and $7 \mathrm{~m}$, the errors between the simulation results and the mean values of the four groups of experimental data are $2.9 \%, 7.5 \%$, and $10.9 \%$, respectively. In point 2 , when the inflating length is $4 \mathrm{~m}, 5.5 \mathrm{~m}$, and $7 \mathrm{~m}$, respectively, the errors between the simulation results and the average values of the four groups of experimental data are $1.9 \%, 9.5 \%$, and $5.4 \%$, respectively. It can be concluded that the maximum error between the simulated results and the average values of the four groups of experimental data is $10.9 \%$ and the minimum error is only $1.9 \%$. When comparing the numerical simulation data with the experimental data, due to the limitation of the numerical simulation conditions, there will be some error with the actual experimental data. Combined with Figure 2, when the inflatable lengths of pipes are $4 \mathrm{~m}, 5.5 \mathrm{~m}$, and $7 \mathrm{~m}$, respectively, the overpressure peak errors obtained by numerical simulation and experiment are all less than $15 \%$, which are within the allowable range of international engineering error standards. The reliability of the LES + ED model used in gas explosion simulation is verified experimentally.

\section{Determination of Effective Length of Intake/ Return Air Roadway}

The intake/return air roadway of the \#415 working face in Chenjiashan coal mine of Shaanxi Province is arranged along the coal seam floor. The length of the $r$ inlet roadway is $1192 \mathrm{~m}$, and the length of the return air roadway is $1170 \mathrm{~m}$. 


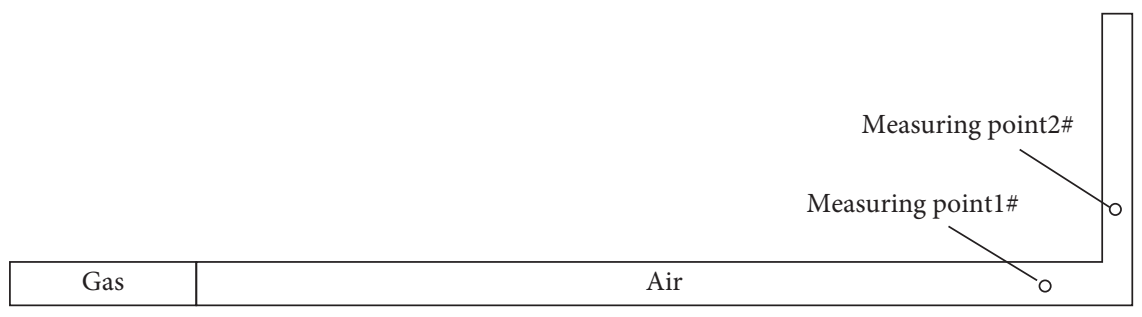

FIGURE 1: Section diagram of physical model.

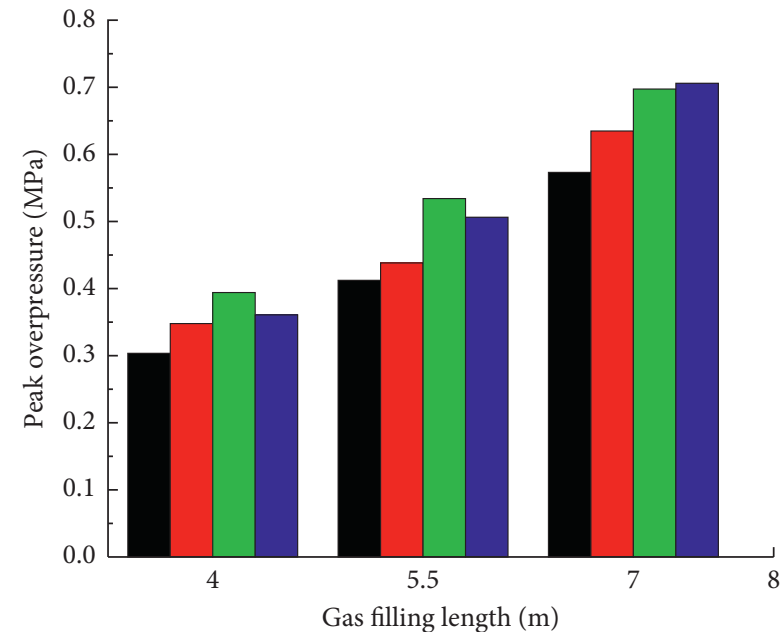

$\square$ Experiment $1 \#$
$\square$ Experiment $2 \#$
$\square$ Experiment 3\#
$\square$ Numerical simulation

(a)

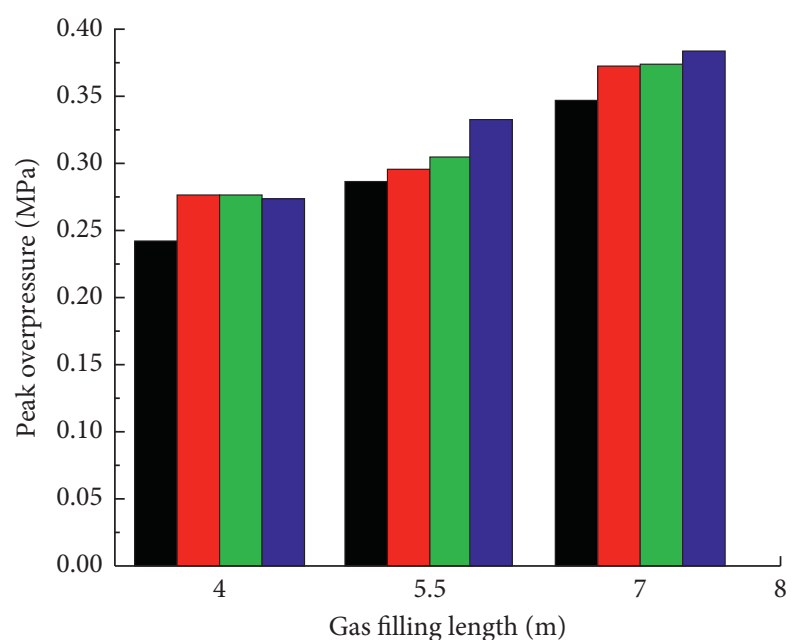

Experiment $1 \#$
Experiment $2 \#$
$\square$ Experiment $3 \#$
numerical simulation

(b)

FIgURE 2: Comparison of CFD simulation and lab experiments results: (a) measuring point 1; (b) measuring point 2.

The cross-section of roadway is a semicircular arch with a parallel arrangement, and the inclined length of the working face is $175 \mathrm{~m}$. In the numerical simulation study, the reasonable length of the intake/return air roadway not only makes the calculation results more accurate but also significantly reduces the workload of numerical calculation. Therefore, the author carried out a simulation study on the gas explosion with U-type ventilation to determine the reasonable effective length of the inlet/outlet roadway.

3.1. Establishing the 3D Model and Meshing. According to the specific parameters of the \#415 working face in Chenjiashan coal mine of Shaanxi Province and the actual situation of the explosion, geometry and mesh modules in workbench are used to establish a three-dimensional physical model and grid division. As shown in Figure 3, the length of the inlet/ return air roadways is $100 \mathrm{~m}$, and the mesh size is $0.5 \mathrm{~m}$. The mesh is refined by local mesh densification technology in the gas filling area and ignition point. As shown in Figure 3, the length of the return air roadway is $100 \mathrm{~m}$, and 9 monitoring points are arranged in the return air roadway, each with a distance of $10 \mathrm{~m}$.
3.2. Initial Conditions and Boundary Conditions. The explosion site of the November 28th major gas explosion accident in Chenjiashan coal mine was the upper corner of the \#415 working face. The inlet and return air outlets are set as pressure outlets, and the others are standard wall surfaces. According to the accident analysis report of the government department of Chenjiashan coal mine gas explosion accident, the mass fractions of $\mathrm{CH}_{4}$ and $\mathrm{O}_{2}$ in the gas filling area of the upper corner are 0.095 and 0.212 , respectively, and the burnt area is a ball with a radius of $0.5 \mathrm{~m}$ in the center of the upper corner. In this area, the mass fraction of $\mathrm{CO}_{2}$ is 0.1456 , the mass fraction of $\mathrm{H}_{2} \mathrm{O}$ is 0.11925 , the standard atmospheric pressure is $101325 \mathrm{~Pa}$, and the temperature is $1600 \mathrm{~K}$. A simple algorithm is used for an iterative solution; nine monitoring points are arranged in the return air roadway, each of which is $10 \mathrm{~m}$ apart.

3.3. Analysis of Numerical Simulation Results. The whole explosion can be regarded as a process that starts from the ignition zone, detonates the gas/air mixture in turn, and propagates from the combustion wave and shock wave to the unburned area. This can be divided into three stages: ignition 


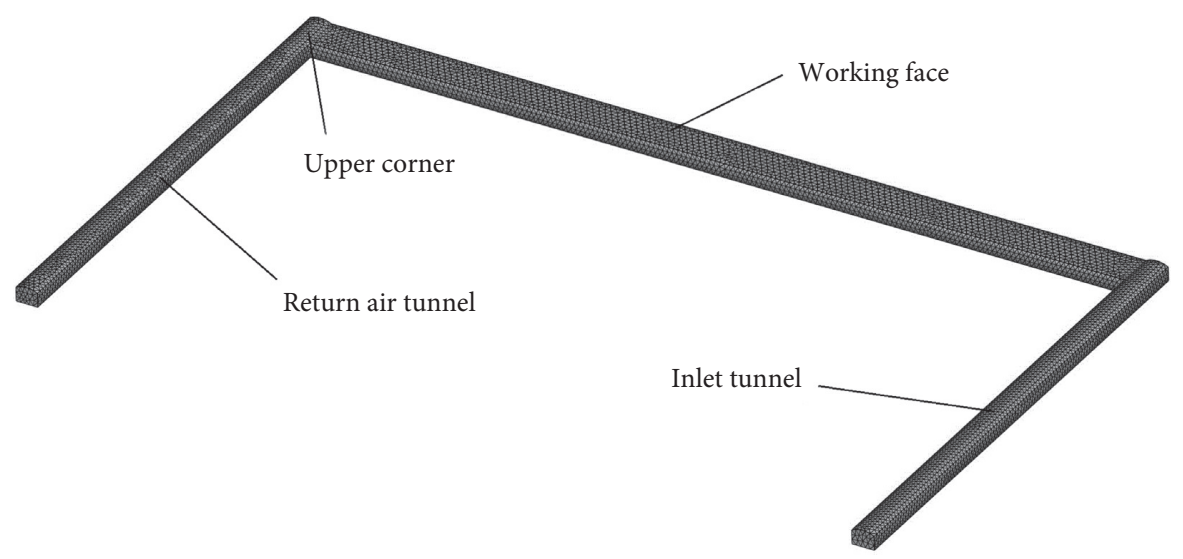

FIgURE 3: Three-dimensional physical model.

stage, compression combustion stage, and single shock wave propagation stage [37]. The propagation cloud diagrams of blast wave with different time scales shown in Figure 4 are given based on simple algorithms and simulation results, respectively, describing the propagation characteristics of blast wave at different moments. The propagation nephogram of the explosion shock wave and the pressure curve of each measuring point are shown in Figures 4 and 5.

The overpressure peak value, peak time, and variation of overpressure peak value between adjacent measuring points and equidistant attenuation coefficient are listed in Table 1.

According to the parameter data in Table 1, the variation of the overpressure peak value and arrival time with distance is shown in Figure 6. The variation of the overpressure peak's $10 \mathrm{~m}$ isometric difference between adjacent measuring points with distance is shown in Figure 7. With an increase in distance, the attenuation coefficient of the overpressure peak and data fitting prediction of each measuring point is shown in Figure 8.

(1) Figure 6 shows that the peak value of overpressure varies greatly within $40 \mathrm{~m}$ from the explosion area. The overpressure peak value of each measuring point basically attenuates linearly beyond $40 \mathrm{~m}$ from the explosion area. The time to reach the peak value increases linearly with an increase in distance.

(2) Figure 7 shows that the difference in the overpressure peak value of each measuring point at measuring points \#1-4 is relatively large, and the difference in the overpressure peak value between adjacent measuring points at measuring points \#4-9 basically fluctuates up and down with a very small amplitude. That is, starting from measuring point \#4 (40 m away from the explosion area), the maximum overpressure attenuation is approximately $0.02 \mathrm{MPa}$ for every $10 \mathrm{~m}$ propagation of the gas explosion shock wave.

(3) Figure 8 shows that the attenuation coefficient of the overpressure peak value of measuring point \#4 (40 m away from the explosion area) to measuring point \#9 (90 $\mathrm{m}$ away from the explosion area) presents an obvious linear increasing relationship, and its functional relationship is $y=0.0066 x+0.1244$.
According to the results in Figures 6-8, the gas explosion shock wave starts to propagate stably in the form of a single shock wave from a distance of $40 \mathrm{~m}$ from the explosion area. Therefore, the length of intake/return air roadway for the gas explosion simulation with U-type ventilation is determined to be $40 \mathrm{~m}$. When the length of $\mathrm{U}$-shaped gas explosion simulation inlet and return air tunnel is determined to be $40 \mathrm{~m}$, we can provide a certain reference for the specific location of explosion-proof door according to the effective length and choose different support modes to ensure the stability of roadway structure. In addition, it not only ensures the accuracy of the calculation results but also greatly reduces the workload of numerical simulation.

\section{Study of Influence of Different Roadway Cross-Section Shapes on Shock Wave Law of Gas Explosion}

According to different mine types, mining methods, geological conditions, hydrological conditions, and other factors, the roadway adopts different sectional shapes. The most commonly used cross-sectional shapes of roadways in China are rectangular, trapezoidal, and semicircular arch. Therefore, in the study of U-shaped ventilation gas explosion with different cross-section shapes, rectangle, trapezoid, and semicircle arch are adopted, respectively, as shown in Figure 9.

4.1. Physical Model and Initial/Boundary Conditions. Three-dimensional physical models of the intake/return air roadway and working face with rectangular, trapezoidal, and semicircular arch cross-sections are established as shown in Figure 10. The vertical height of the roadway is $0.3 \mathrm{~m}$ higher than that of the working face when the cross-sectional shape is a semicircular arch, and the roadway height is the same as that of the working face when the cross-sectional shape is rectangular or trapezoidal. The length of the inlet/return air roadway is $40 \mathrm{~m}$. The initial and boundary conditions of the numerical simulation are the same as those in Section 3.2. 


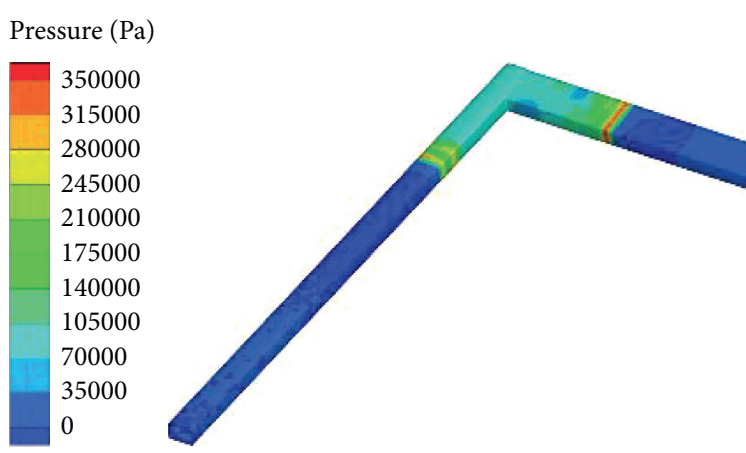

(a)

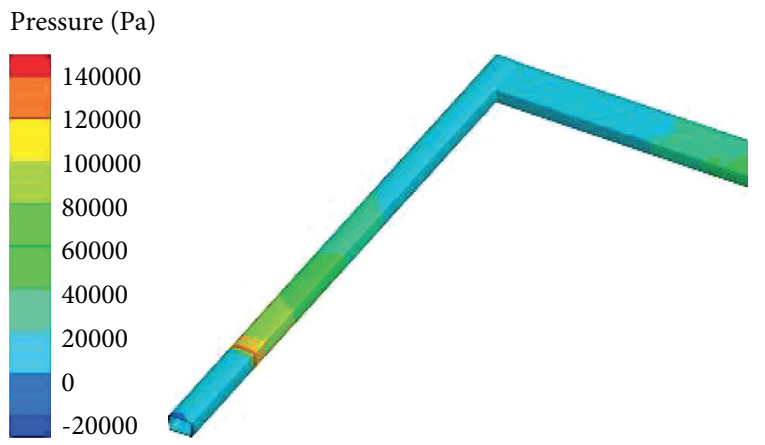

(c)

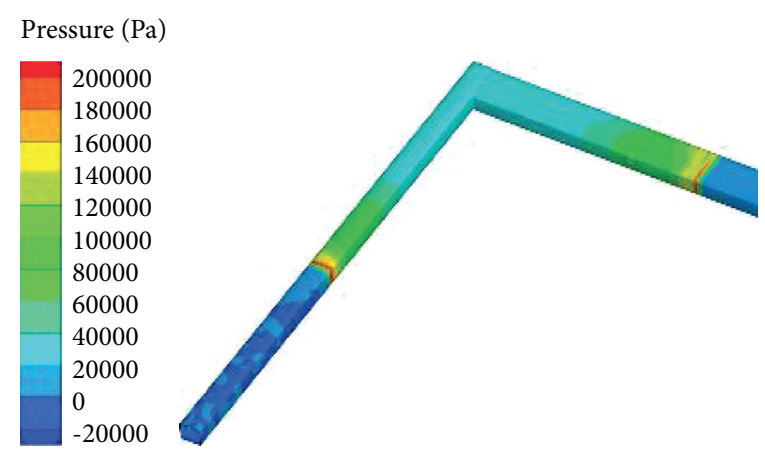

(b)

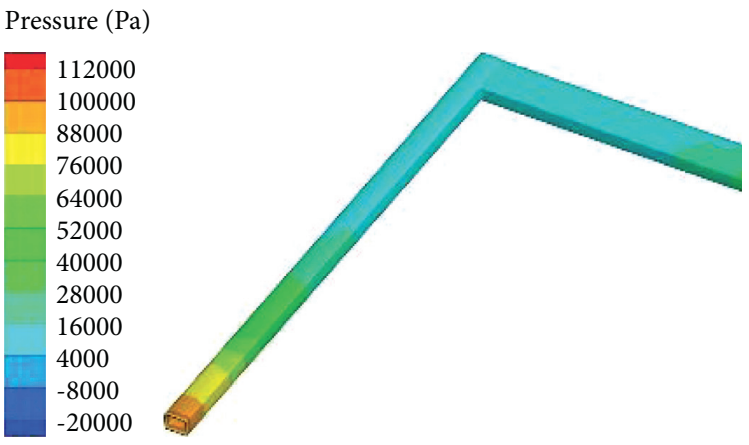

(d)

Figure 4: Cloud diagram of shock wave propagation at each moment: (a) $t=33.5 \mathrm{~ms}$; (b) $t=88 \mathrm{~ms}$; (c) $t=140 \mathrm{~ms}$; (d) $t=181 \mathrm{~ms}$.

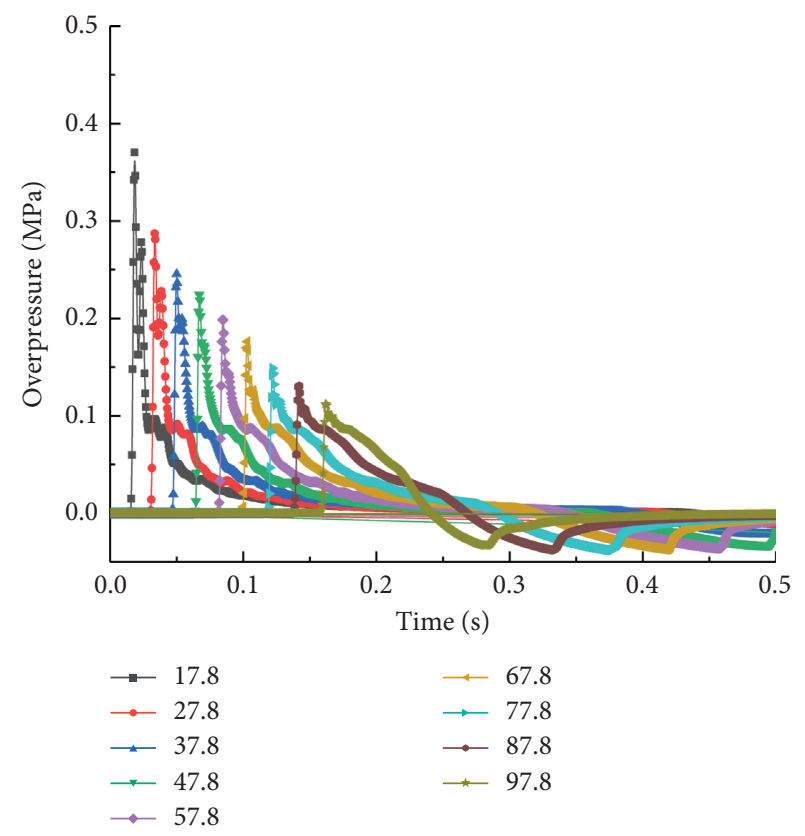

FIGURE 5: Overpressure change graph of each measuring point.

4.2. Analysis of Numerical Simulation Results. The numerical simulation of different cross-section shapes of roadway is carried out, the upper-corner gas is detonated by high temperature ignition point, the rectangular section is taken as an example, and the pressure nephogram of gas explosion shock wave is obtained at different times as shown in
Figure 11. The pressure cloud image of gas explosion shock wave at typical time was selected to better explain the propagation characteristics of explosion shock wave in the upper corner of the U-shaped ventilation model.

According to the above pressure nephogram of gas explosion shock wave at different times, it can be seen that after the gas explosion occurs in the upper corner of U-type ventilation mode, the shock wave first propagates through the return air roadway and coal mining face, and the shock wave pressure gradually attenuates in the process of propagation. When the shock wave propagates to the corner where the working face is connected with the air inlet roadway, it can be seen that the shock wave is reflected and superimposed, which causes the overpressure of the shock wave to rise in a very short time.

The data of monitoring points are sorted out to get the change of overpressure at each point of $40 \mathrm{~m}$ inlet/return air roadway with different cross-section shapes, as shown in Figures 12 and 13.

Figures 12 and 13 show that when the upper-corner gas explosion occurs, the initial overpressure of the inlet/return air roadways with different cross-sectional shapes is different. When the cross-sectional shape is a trapezoid, the initial overpressure of the explosion shock wave propagating to the inlet/return air roadway is the largest, followed by the rectangular shape, and the minimum occurs with the semicircular arch. When the explosion shock wave propagates in roadways of different cross-sectional shapes, it is consistent with the initial overpressure at all positions in the roadway. The results show that the maximum overpressure 
TABLE 1: Parameters of each monitoring point.

\begin{tabular}{lccccccccc}
\hline Monitoring point & Point 1\# & Point 2\# & Point 3\# & Point 4\# & Point 5\# & Point 6\# & Point 7\# & Point 8\# & Point 9\# \\
\hline Peak overpressure (MPa) & 0.370 & 0.287 & 0.245 & 0.224 & 0.199 & 0.176 & 0.149 & 0.131 & 0.109 \\
Peak time (s) & 0.018 & 0.033 & 0.050 & 0.067 & 0.087 & 0.103 & 0.122 & 0.142 & 0.162 \\
Peak value difference (MPa) & & 0.083 & 0.042 & 0.021 & 0.025 & 0.023 & 0.027 & 0.018 & 0.022 \\
Attenuation coefficient K & & 0.224 & 0.338 & 0.395 & 0.462 & 0.524 & 0.597 & 0.646 & 0.705 \\
\hline
\end{tabular}

Note: (1) the peak value difference is the overpressure peak value difference between adjacent measuring points and (2) attenuation coefficient $\mathrm{K}$ is the reduction rate of each measuring point relative to measuring point $1 \#$.

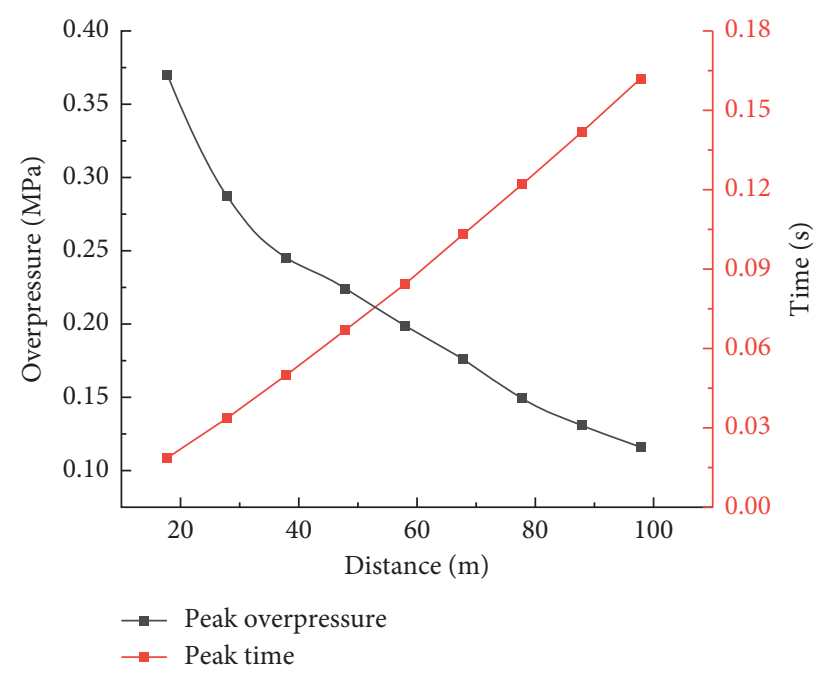

FIgURe 6: Peak overpressure and peak time change with distance.

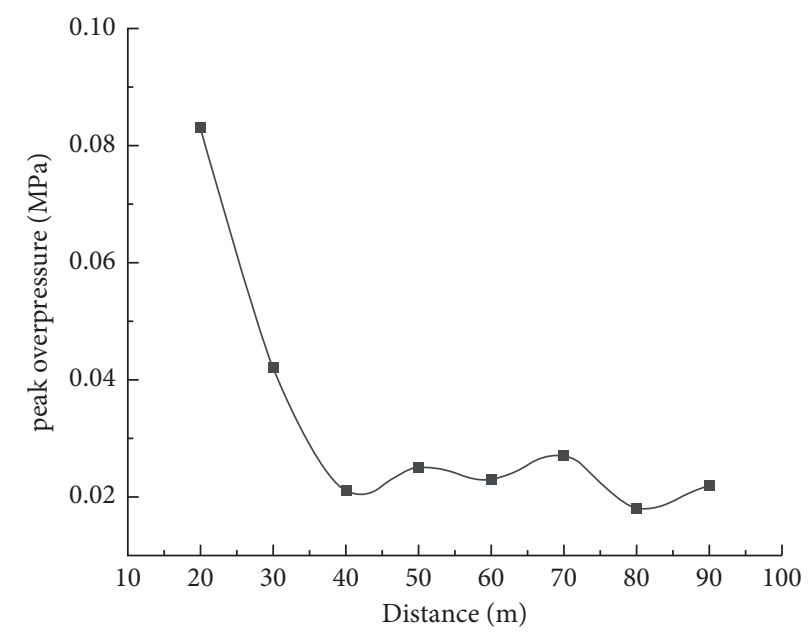

FIGURE 7: Overpressure difference between adjacent measuring points.

occurs with the trapezoidal shape, the second is rectangular, and the smallest is the semicircular arch. However, the attenuation trend of overpressure propagation is the same for all three cross-sectional shapes.

In the three-dimensional physical models of the above three roadway cross-sectional shapes, the size of the coal mining face is always consistent with the actual situation. Under the conditions of different cross-sectional shapes of the inlet/return air roadway, the overpressure changes at various working face position, which is shown in Figure 14.
Figure 14 shows that under the condition of different cross-sectional shapes of air inlet/return roadways, the initial overpressure of the working face cut is largest when the cross-sectional shape of the roadway is rectangular, followed by the semicircular arch and the trapezoid. With the propagation of the shock wave along the working face cut, the farther away from the upper corner of different crosssectional shapes, the closer the overpressure at each point of the cut. The pressure attenuation trend is in line with the power function law. The overpressure attenuation model of 


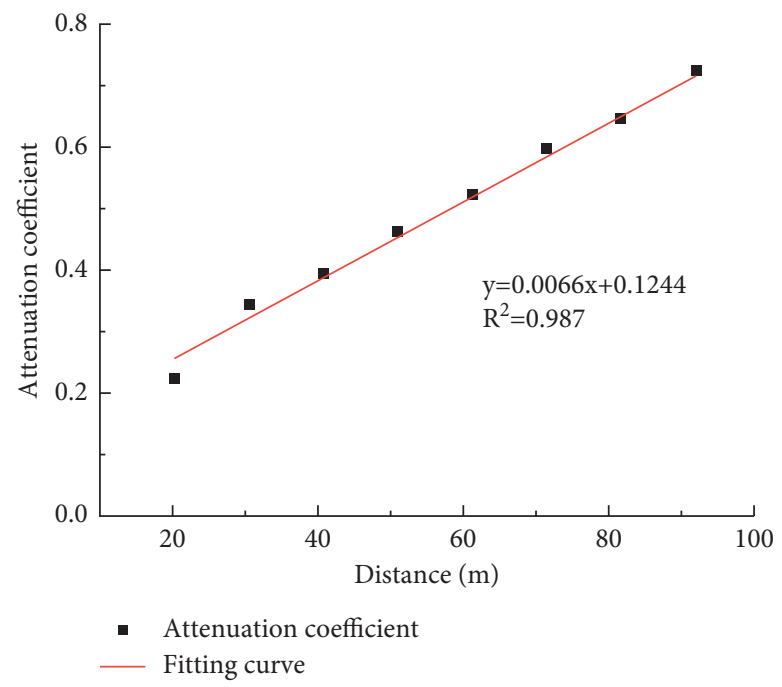

FIgURE 8: Attenuation coefficient varying with distance.
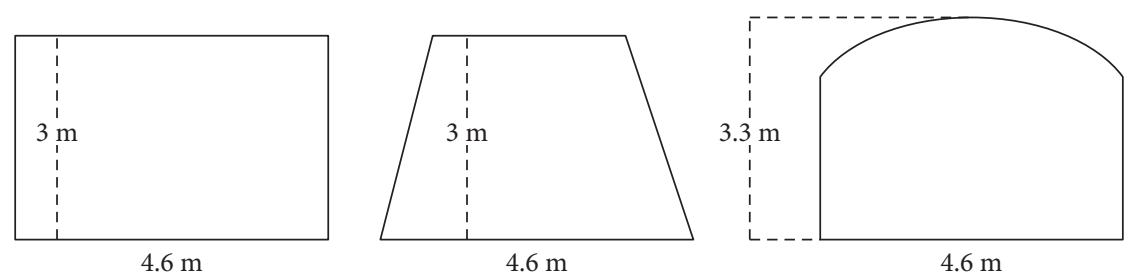

FIgURE 9: Different cross-sectional shapes.

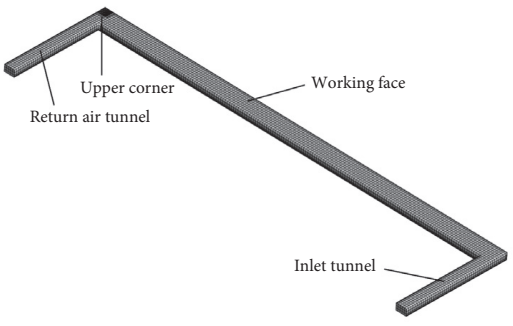

(a)

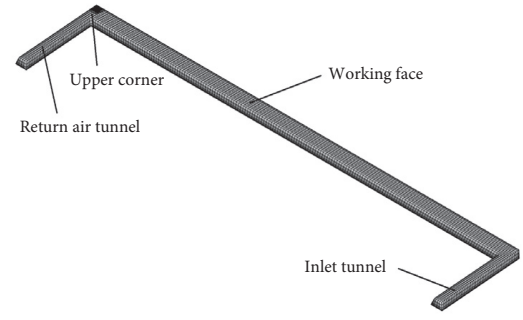

(b)

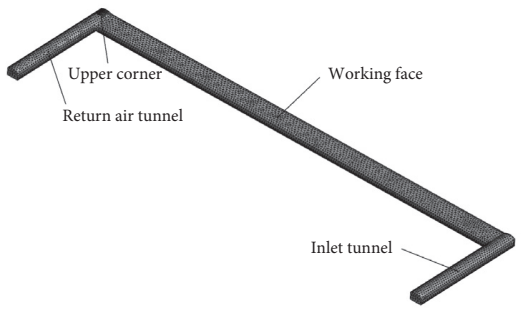

(c)

Figure 10: Physical models of different cross-sectional shapes: (a) rectangular section; (b) trapezoidal section; (c) semicircular arch section.

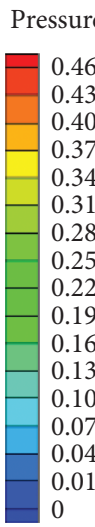

Pressure $(\mathrm{MPa})$

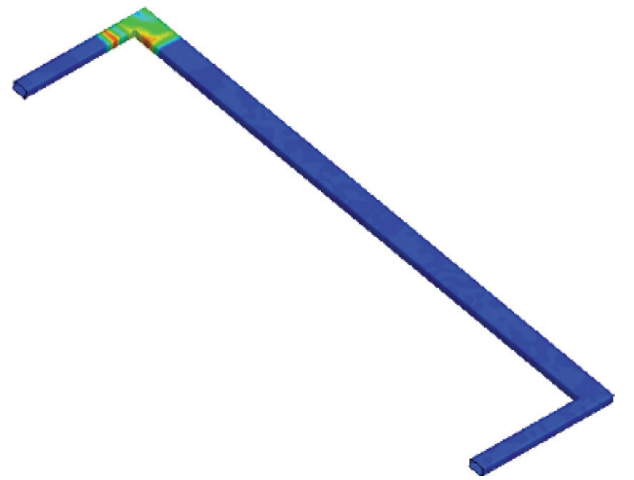

(a)

\begin{tabular}{|l|}
\hline 0.16 \\
0.15 \\
0.14 \\
0.13 \\
0.12 \\
0.11 \\
0.10 \\
0.09 \\
0.08 \\
0.07 \\
0.06 \\
0.04 \\
0.02 \\
0 \\
\hline-0.01 \\
-0.02 \\
-0.03
\end{tabular}

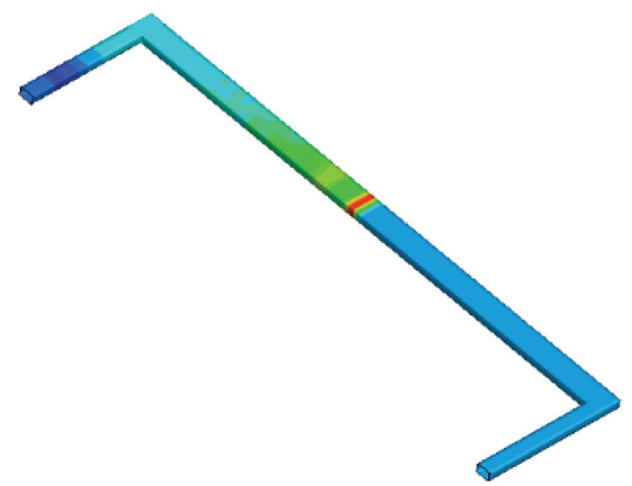

(b)

Figure 11: Continued. 
Pressure $(\mathrm{MPa})$
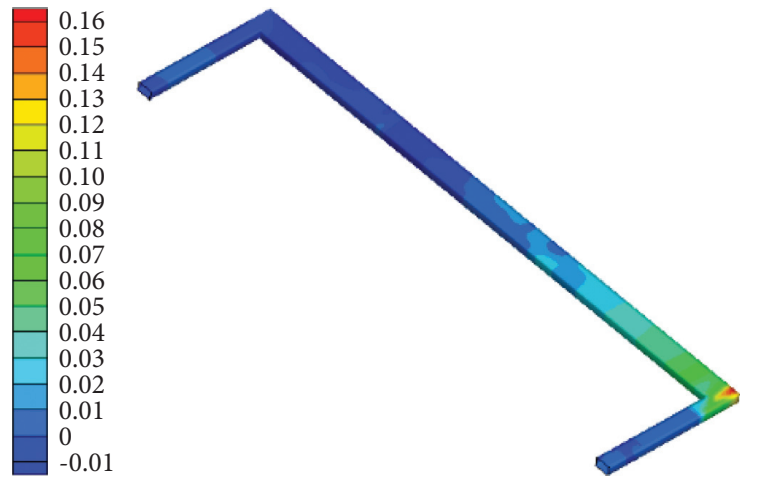

(c)

Pressure (MPa)

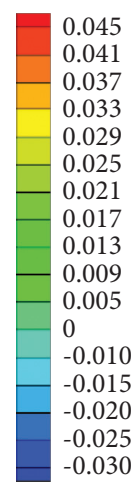

Pressure (MPa)
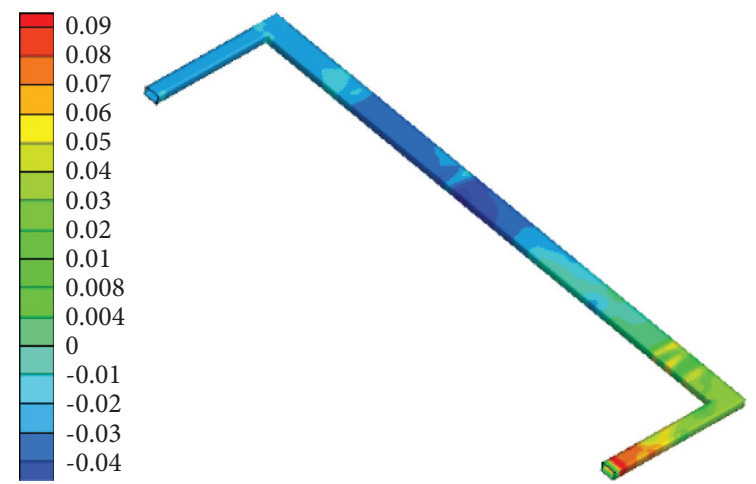

(d)

Pressure (MPa)

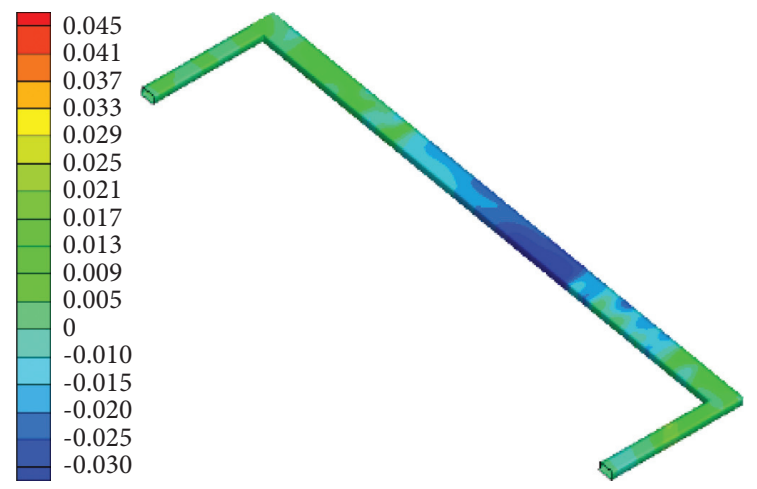

(f)

Figure 11: Pressure nephogram of shock wave at different times (rectangle): (a) $t=0.02 \mathrm{~s}$; (b) $t=0.15 \mathrm{~s}$; (c) $t=0.36 \mathrm{~s}$; (d) $t=0.44 \mathrm{~s}$; (e) $t=0.6 \mathrm{~s}$; (f) $t=0.94 \mathrm{~s}$.

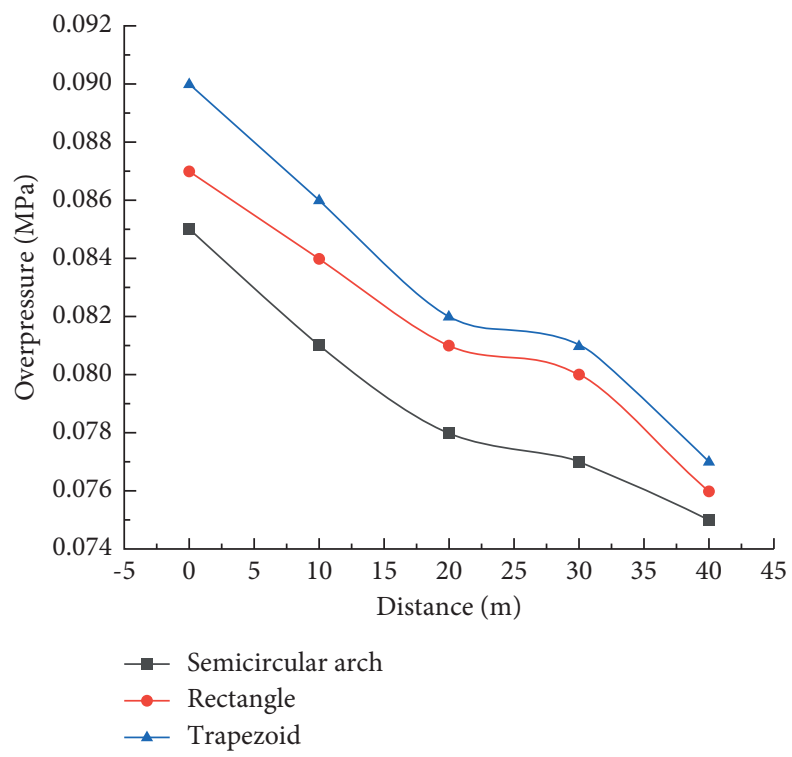

FIGURE 12: Pressure changes of air inlet tunnel with different cross-sectional shapes. 


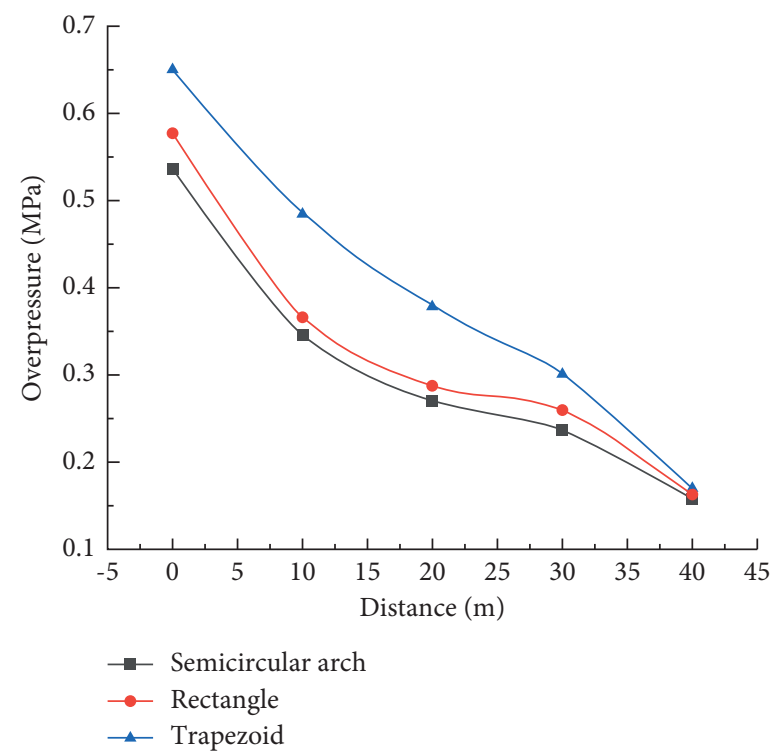

FIgURE 13: Pressure change of return air tunnel with different cross-sectional shapes.

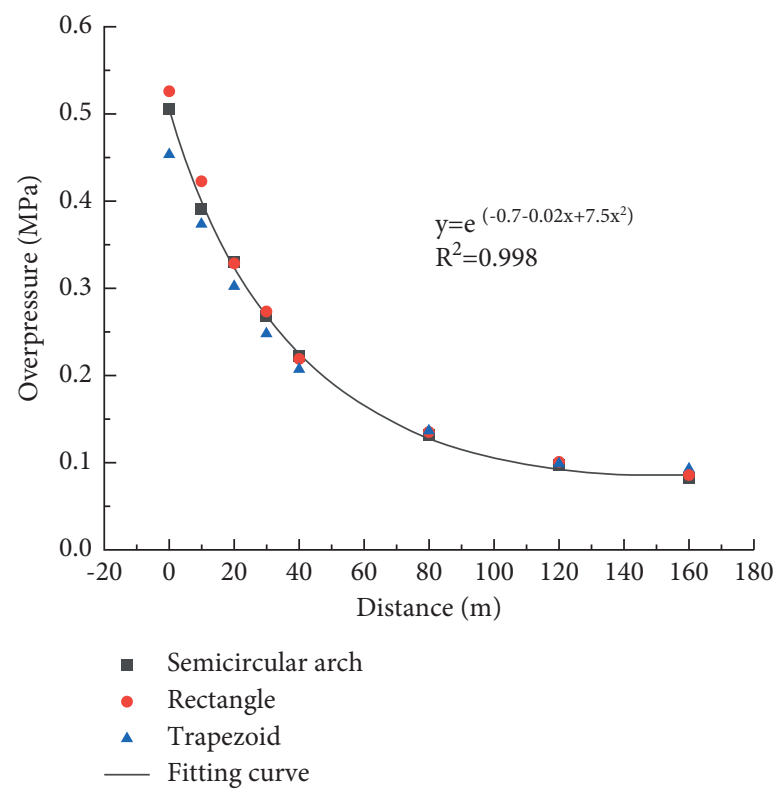

FIGURE 14: Changes of overpressure in the cut eye of the working face with different cross-sectional shapes.

the upper-corner gas explosion working face is obtained as follows: $y=e^{(-0.7-0.02 x+7.5 x \hat{2})}$.

\section{Conclusions}

(1) Through an analysis of the attenuation coefficient and overpressure difference of the adjacent measuring points, it is concluded that the blast wave of a gas explosion propagates stably in the form of a single shock wave at $40 \mathrm{~m}$ away from the explosion source, and the effective length of the inlet/return air roadway in the upper corner with $U$-type ventilation is determined to be $40 \mathrm{~m}$.
(2) The simulation results of a gas explosion in the upper corner of three different cross-sectional shapes were analyzed. When the cross-sectional shape is a trapezoid, the initial overpressure of the explosion shock wave propagating to the inlet/return air roadway is the largest, followed by the rectangle and semicircle arch, but the attenuation trend of overpressure propagation in the three different crosssectional shapes is the same.

(3) When the gas explosion shock wave propagates along the cut hole of the working face, the farther the distance from the upper-corner angle, the closer the overpressure at each point of the cut hole. The decay 
trend of the overpressure conforms to the law of power function. The attenuation model is $y=e^{(-0.7-0.02 x+7.5 x \hat{2})}$.

\section{Data Availability}

The data used to support the findings of this study are included within the article.

\section{Conflicts of Interest}

The authors declare that they have no conflicts of interest.

\section{Acknowledgments}

This work was supported by National Natural Science Foundation of China (52074106 and 51734007). This project was also supported by Zhongyuan Postdoctoral Innovative Talent (ZYQR201810171), China Postdoctoral Science Foundation (2019M652536), and Henan Postdoctoral Foundation (001801016) and funded by the Doctoral Fund of Henan Polytechnic University (B2018-59).

\section{References}

[1] A. L. Both, G. Atanga, and H. Hisken, "CFD modelling of gas explosions: optimising sub-grid model parameters," Journal of Loss Prevention in the Process Industries, vol. 60, pp. 159-173, 2019.

[2] B. Y. Jing, B. Q. Lin, S. L. Shi, C. J. Zhu, and Z. W. Li, "Theoretical analysis on the attenuation characteristics of strong shock wave of gas explosion," Procedia Engineering, vol. 24, pp. 422-425, 2011.

[3] M. Gieras, R. Klemens, G. Rarata, and P. Wolański, "Determination of explosion parameters of methane-air mixtures in the chamber of $40 \mathrm{dm} 3$ at normal and elevated temperature," Journal of Loss Prevention in the Process Industries, vol. 19, no. 2-3, pp. 263-270, 2006.

[4] M. Scheid, A. Geiler, and U. Krause, "Experiments on the influence of pre-ignition turbulence on vented gas and dust explosions," Journal of Loss Prevention in the Process Industries, vol. 19, no. 2-3, pp. 194-199, 2006.

[5] H. T. Li, X. K. Chen, J. Deng et al., "CFD analysis and experimental study on the effect of oxygen level, particle size, and dust concentration on the flame evolution characteristics and explosion severity of cornstarch dust cloud deflagration in a spherical chamber," Powder Technology, vol. 372, 2020.

[6] S. Wang, Z. Shi, X. Peng et al., "Effect of the ignition delay time on explosion severity parameters of coal dust/air mixtures," Powder Technology, vol. 342, pp. 509-516, 2019.

[7] B. W. Lei, B. Wu, Y. T. Zhao, and M. A. Ashraf, "Numerical simulation and experimental study on formation of high concentration of h2 generated by gas explosion," Polish Maritime Research, vol. 23, 2016.

[8] Q. Liu, Y. Zhang, F. Niu, and L. Li, "Study on the flame propagation and gas explosion in propane/air mixtures," Fuel, vol. 140, pp. 677-684, 2015.

[9] Y. Xu, Y. M. Huang, and G. W. Ma, "A review on effects of different factors on gas explosions in underground structures," Underground Space, vol. 5, pp. 298-314, 2019.

[10] M. Yu, K. Zheng, and T. Chu, "Gas explosion flame propagation over various hollow-square obstacles," Journal of
Natural Gas Science and Engineering, vol. 30, pp. 221-227, 2016.

[11] G. X. Jing, Y. L. Wu, S. S. Guo, H. Y. Shao, C. Liu, and S. Q. Zhang, "The influence of obstacles on the flame propagation law of gas and coal dust explosion," Journal of Safety Science and Technology, vol. 15, no. 9, pp. 99-104, 2019.

[12] P. F. Pan, Y. X. Tan, and Z. Q. Wang, "Study on the influence of strip obstacles on gas explosion characteristics," Journal of Safety Science and Technology, vol. 6, no. 5, pp. 71-76, 2010.

[13] F. H. Wang, W. Chen, X. P. Wen, W. L. Zhao, and Z. C. Liu, "Numerical simulation and mechanism analysis of gas explosion suppression by ultrasonic water mist," Energy Sources Part A Recovery Utilization \& Environmental Effects, vol. 41, pp. 1-13, 2019

[14] H. Shao, S. Jiang, X. Zhang, Z. Wu, K. Wang, and W. Zhang, "Influence of vacuum degree on the effect of gas explosion suppression by vacuum chamber," Journal of Loss Prevention in the Process Industries, vol. 38, pp. 214-223, 2015.

[15] K. Wang, S. Jiang, X. Ma, Z. Wu, W. Zhang, and H. Shao, "Study of the destruction of ventilation systems in coal mines due to gas explosions," Powder Technology, vol. 286, pp. 401-411, 2015.

[16] C. Wang, Y. Zhao, and E. K. Addai, "Investigation on propagation mechanism of large scale mine gas explosions," Journal of Loss Prevention in the Process Industries, vol. 49, pp. 342-347, 2017.

[17] A. H. Yan, B. S. Nie, L. C. Dai et al., "Numerical simulation on the gas explosion propagation related to roadway," Procedia Engineering, vol. 26, pp. 1563-1570, 2011.

[18] Z. A. Huang, Z. G. Liu, Y. S. Zhang, and Y. S. Zhang, "Numerical simulation and study on the transmission law of flame and pressure wave of pipeline gas explosion," Safety Science, vol. 50, no. 4, pp. 806-810, 2012.

[19] W. Cao, W. Gao, Y. Peng, J. Liang, F. Pan, and S. Xu, "Experimental and numerical study on flame propagation behaviors in coal dust explosions," Powder Technology, vol. 266, pp. $456-462,2014$.

[20] C. J. Zhu, B. Q. Lin, B. Y. Jiang, Q. Liu, and Y. D. Hong, "Numerical simulation of blast wave oscillation effects on a premixed methane/air explosion in closed-end ducts," Journal of Loss Prevention in the Process Industries, vol. 26, no. 4, pp. 851-861, 2013.

[21] Q. Zhang, L. Pang, and H. M. Liang, "Effect of scale on the explosion of methane in air and its shockwave," Journal of Loss Prevention in the Process Industries, vol. 24, no. 1, pp. 43-48, 2011.

[22] J. Li and H. Hao, "Numerical and analytical prediction of pressure and impulse from vented gas explosion in large cylindrical tanks," Process Safety and Environmental Protection, vol. 127, pp. 226-244, 2019.

[23] Q. Ye, B. Q. Lin, Z. Z. Jia, and C. J. Zhu, "Propagation law and analysis of gas explosion in bend duct," Procedia Earth and Planetary Science, vol. 1, no. 1, pp. 316-321, 2009.

[24] Q. Y. Yang, B. M. Shi, and L. L. Zhang, "Influence of turning angle on coal dust explosion induced by gas explosion," China Safety Science Journal, vol. 29, no. 7, pp. 58-63, 2019.

[25] B. J. Xie, Y. J. Du, and L. Wang, "Experiment and numerical simulation of flame propagation law of gas explosion in bifurcated pipe," Journal of Chongqing University, vol. 42, no. 6, pp. 69-77, 2019.

[26] W. B. Pang, Y. C. Li, and X. He, "Experimental study on arrival time of chemical explosion shock wave in T-channel," Explosion and Shock Waves, vol. 1, pp. 63-67, 2007. 
[27] C. Zhai, B. Q. Lin, Q. Ye, Z. G. Lu, C. J. Zhu, and Z. Y. Zhang, "Influence of abnormal pipeline structure on gas explosion propagation characteristics," Journal of Xi'an University of Science and Technology, vol. 2, pp. 274-278, 2008.

[28] Y. Guo, C. Liu, D. Wang, and R. He, "Numerical study and safety spacing of buried parallel gas pipelines: a study based on TNT equivalent method," International Journal of Pressure Vessels and Piping, vol. 168, pp. 246-257, 2018.

[29] C. J. Zhu, B. Q. Lin, B. Y. Jiang, and C. Zhai, "Propagation characteristics of flame and shock wave of gas explosion in parallel roadway network," Journal of China University of Mining \& Technology, vol. 40, no. 3, pp. 385-389, 2011.

[30] R. C. Liu and Y. F. Zhu, "Full scale simulation of gas explosion in long-wall face of coal mine," China Safety Science Journal, vol. 28, no. 12, pp. 58-64, 2018.

[31] B. Y. Jiang, B. Q. Lin, C. J. Zhu, C. M. Shen, and C. Zhai, "Numerical simulation of propagation characteristics of gas explosion shock wave in roadway network of coal mining face," Journal of China Coal Society, vol. 36, no. 06, pp. 968-972, 2011.

[32] Z. G. Liu, Study on Detailed Chemical Reaction Mechanism Acceleration Algorithm for Large Eddy Simulation of Turbulent Combustion, University of Chinese Academy of Sciences (Institute of Engineering Thermophysics), Beijing, China, 2019.

[33] X. Wen, M. Xie, M. Yu, and Z. Liu, "Dynamic characteristics of gas deflagration in small-scale confined space," Combustion Science and Technology, vol. 19, no. 4, pp. 347-351, 2013.

[34] X. Wen, M. Yu, H. Deng, and J. Chen, "Large eddy simulation of gas turbulence deflagration in small-scale confined space," Ciesc journal, vol. 67, no. 5, pp. 1837-1843, 2016.

[35] Z. Li, M. Yu, and W. Ji, "Numerical simulation of turbulent flame induced by obstacles in gas explosion," Journal of henan polytechnic university (natural science edition), vol. 34 , no. 2 , pp. 167-170, 2015.

[36] G. X. Jing, Z. W. Jia, L. Cheng, Z. W. Duan, H. Li, and Z. Y. Gao, Propagation Law and Damage Model of Gas Explosion under Complex Conditions, Science Press, Beijing, China, 2017.

[37] J. D. Xu, S. L. Xu, and G. Y. Yang, "Experimental study on propagation of mine gas explosion," Coal Science and Technology, vol. 7, pp. 55-57, 2004. 